\title{
A Practical Methodology for Development of a Network of e-Government Domain Ontologies
}

\author{
Hele-Mai Haav \\ Institute of Cybernetics at Tallinn University of Technology, Akadeemia 21, \\ 12618 Tallinn, Estonia \\ helemai@cs.ioc.ee
}

\begin{abstract}
Ontologies are widely proposed for solving semantic interoperability problems in e-government domain. However, less attention is paid on methodological issues of ontology development in this domain. These are not sufficiently solved. Existing approaches in ontology development are technically very demanding and require long learning curve and are therefore inefficient for this purpose. E-government domain ontologies could be efficiently developed by domain experts rather than ontology engineers. The paper proposes a novel ontology development methodology for construction of light-weight domain ontologies by bringing ontology modeling closer to domain experts that are actual domain knowledge holders. The approach is illustrated and evaluated by development of a network of e-government domain ontologies used for achieving semantic interoperability of state information systems (IS) in Estonia.
\end{abstract}

Keywords: ontology, ontology networks, ontology development methodology, e-government.

\section{Introduction}

Applicability of semantic technologies in the e-Government domain has been investigated for many years. A number of EU projects (e.g. SemanticGov [11], SmartGov [3], OntoGov [14], Access-eGOV [2], etc) are devoted to the several aspects of using ontologies in e-government domain and for different applications. Most of the projects are dealing with building semantic e-government services (i.e. electronic governmental services enhanced by semantic annotations) in order to provide their semantic interoperability.

Many member states of the European Union have started some semantic interoperability initiatives as for example Italian's initiative in public administration ${ }^{1}$, Finnish semantic initiative FinnONTO [7], Semantic Latvia project [1] and Estonian Semantic Interoperability Framework $[6,15]$.

There are also pan-European initiatives, which include SEMIC (SEMantic Interoperability Centre Europe) ${ }^{2}$ and SemanticGov project [11].

\footnotetext{
${ }^{1}$ http: / /www.digitpa.gov.it

${ }^{2}$ http://semic.eu/about_semantic_interoperability_centre_europe.html
} 
However, practical effects of this research and initiatives to e-government domain and applications do not well meet expectations.

One of the main reasons as three years experience of Estonian semantic interoperability initiative shows is complexity of the semantic modeling (e.g. ontology development) and annotation process. There is lack of ontology development methodologies that are not technically demanding in order to be used by e-government domain experts and that are easy to learn by employees of public administrations (PA-s).

This paper concentrates to the problem of complexity of semantic modeling by answering the question: How to easily develop domain ontologies that could be used for semantic annotation of e-government data services?

Majority of current ontology development methodologies $[4,5,13,16]$ require a role of mediator (e.g. knowledge engineer) between actual knowledge holders (e.g. domain experts) and ontology engineers who encode knowledge in one of available onotology formalisms (e.g. $\mathrm{OWL}^{3}$ ).

In contrast, this paper proposes a novel ontology development methodology for construction of light-weight domain ontologies by bringing ontology modeling closer to domain experts (PA employees) that are actual knowledge holders. The methodology could be used for construction of domain ontologies by domain experts without any high level knowledge of semantic technologies.

The approach is illustrated and evaluated by development of network of egovernment domain ontologies used for annotation of governmental data services in order to support semantic interoperability of state information systems (IS) in Estonia.

The paper is structured as follows. Section 2 gives a brief overview of related semantic e-government projects and ontology creation methodologies. In section 3 a motivating Estonian e-government case study is described and analysed. Section 4 presents a practical ontology development methodology for network of e-government domain ontologies. Section 5 is evaluation of the methodology and section 6 concludes the work.

\section{Related Works}

Majority of e-government projects use ontologies for achieving semantic interoperability and integration between e-Government systems. For example, in OntoGov [14] ontologies are constructed for representing the meaning of processed data, resources and provided functionality of e-Government services. However, it is not always clear, what methodology is used for the ontology development in egovernment projects. Usually top-down approach is used. There are some general methodological approaches (e.g., METHONTOLOGY [4], On-To-Knowledge [13], etc.) for ontology construction. More detailed overview of wide range of methodologies can be found in [4].

${ }^{3}$ OWL DL, www.w3 . org/TR/owl-guide 
The most well-know methodology is METHONTOLOGY methodology [4] that enables the construction of ontologies at the knowledge level. METHONTOLOGY is based on the main activities identified by the software development process [8] and by knowledge engineering methodologies. This methodology includes the ontology development process, a life cycle based on evolving prototypes and techniques to carry out each activity in the management, development and support activities. Another approach is TOVE [16] where authors suggest using questionnaires, which is useful where domain experts have very little knowledge of knowledge modeling.

There are also attempts to improve existing methodologies by incorporating Rapid Application Development (RAD) approaches in ontology development [10, 17].

The NeOn [5] ontology development methodology takes software engineering approach and goes step further having an ambitious goal to serve as a methodology for building a very large number of ontologies embedded in ontology networks built collaboratively by distributed teams. NeOn methodology is also supported by several tools collected into the NeOn toolkit [5].

All these approaches have turned ontology construction to an engineering activity.

However, there is lack of methodologies that follow a user-oriented (e.g. domain expert oriented) approach. The majority of approaches require extensive technical knowledge of formal languages and techniques for capturing knowledge of a domain.

Therefore, this paper is devoted to bringing ontology modeling closer to domain experts. The proposed practical methodology for e-government ontology building is taking into account some of the proposals from the NeOn methodology as egovernment ontologies in principle are networked ontologies.

\section{Use Case: Semantic Interoperability of e-Government Services in Estonia}

In Estonia, interoperability of state IS-s is achieved by using middleware platform XRoad [9] that provides the technical interoperability of the components and registries of state IS. X-Road is a technical and organizational environment that enables secure data transfer between digital government databases and enables secure data transfer between individuals and government institutions. X-road platform is based on SOAP standard and governmental e-service descriptions are presented in $\mathrm{WSDL}^{5}$ language. According to the statistics of the using X-Road [9] the estimated number of uses in 2010 was 100 million.

The X-Road does not support semantic interoperability. In order to initiate semantic interoperability activities, Ministry of Economic Affairs issued strategic document on semantic interoperability of state information technology (IT) in 2005 . This document presents a vision of semantic interoperability of state information systems in Estonia. Following this vision, the semantic interoperability architecture for Estonian state IS-s was proposed in 2007 [6]. It is designed as the semantic layer to be built on top of X-Road infrastructure.

\footnotetext{
${ }^{4}$ SOAP standard, http: / / www .w3 .org/TR/soap12-part1/\#intro

${ }^{5}$ WSDL, http : / /www.w3 . org/TR/wsdl
} 
Important components of semantic interoperability architecture are domain ontologies of state IS-s. Primary use of ontologies as foreseen by the architecture is semantic annotation of descriptions of e-government data services and data structures of databases of state IS-s. As WSDL descriptions of existing services are available then SAWSDL $^{6}$ has been chosen for enabling these to be enhanced with semantic descriptions. For data structures, their $\mathrm{XMI}^{7}$ descriptions are enriched with SAWSDL references to corresponding components of domain ontologies.

As a repository of ontologies and semantic descriptions, the Administrative System of the State Information System (RIHA) [12] is used. It is a secure web-based database and software application that supports various processes of public sector information systems and that stores systematic and reliable metadata about public databases. According to statistics [12], at the end of 2009, RIHA contained the data of 450 databases and IS-s.

Another important tool currently under development is SEHKE [15] that is a webbased collaborative environment for creating, managing and annotating semantic resources of state IS-s and registries.

The semantic interoperability architecture was set up and supported by related legislation in Estonia in 2008 demanding from holders of state IS-s creation of corresponding domain ontologies and semantic annotations of web-services. However, in reality this is not happening so easily. There is lack of knowledge about semantic technologies in general and about ontology engineering in particular. Within the semantic interoperability framework a large number of training courses have been provided to stakeholders (i.e. approximately to 200 participants), mainly to domain experts, who are responsible for ontology creation in their respective domains. A feedback from the very first training courses already has shown a need for a methodology and guidelines for ontology development that are closer to domain experts than general existing methodologies. In public administration agencies, it was impossible to have a large number of ontology engineers or knowledge engineers to help to convert domain knowledge to a formal ontology. The practical methodology for development of e-government domain ontologies presented in this paper is motivated by the situation described above.

\section{A Practical Ontology Development Methodology}

\subsection{Goal, Object and Approach of the Methodology}

A practical ontology development methodology presented in this paper aims at providing a systematic approach to development of ontologies capturing domain knowledge and semantics of databases fields related to different state IS-s. Most important goal of ontology development is their reuse across many applications and for building other ontologies.

\footnotetext{
${ }^{6}$ SAWSDL, http: / /www.w3 .org/TR/sawsdl/

${ }^{7}$ XMI, http: / / www . omg . org/spec/XMI /
} 
From the methodological point of view, development of ontologies is a complex task and a lot of ontology building experiences are needed in order to produce well established ontologies. Therefore, one of the goals of current methodology is to make ontology development process easier for domain experts by providing processes and guidelines that they could use.

The provided methodology is primarily intended to be used for development of ontologies represented in OWL enabling semantic annotation of database objects and e-government services (described in WSDL) of state IS-s.

In reality, domain ontologies of state IS-s form a network of ontologies, in what different domain ontologies are related by linking, modularity, versioning or by other relationships. Such ontology network creates certain requirements to ontology development methodology. Ontology maintenance becomes much more complex in the case of ontology network than in the case of isolated ontologies.

Concerning methods for building domain ontologies, already accepted ontology development methodologies like METHONTOLOGY [4] and NeOn [5] are taken as a basis for creation of a new methodology that better meets specific needs of domain experts.

\subsection{Ontology Development Process}

The ontology development process refers to activities that are performed when building ontologies as defined in METHONTOLOGY methodology for ontology construction [4]. Ontology development process proposed in this paper is based on this definition. It takes into account as an input different re-useable knowledge resources that are available in governmental agencies, who are responsible of their IS as well as resources managed by developers of those IS. For example, re-useable knowledge resources could be regulations of creation of a state IS, conceptual schema of a state IS database, data structures in the form of XMI file, descriptions of data services etc.

For creation of a domain ontology for each of the state IS domains an iterative life cycle model was chosen. According to this model ontology development starts from partial satisfaction of requirements. During each of the iterations ontologies will be improved until all the requirements will be met. This life cycle model was successfully used in development of many domain ontologies of Estonian state IS including the most important and complex ones as ontology of central commercial register $^{8}$, address objects, mineral deposits, Estonian topographic database etc. In development of a network of domain ontologies of state IS a method of evolutionary prototyping is proposed. According to this a partial result (a partial network of state IS domain ontologies) that meets known requirements is developed at first. This prototype of ontology network is evaluated and assessed by using it in different applications and the requirements are refined based on such evaluations.

\footnotetext{
${ }^{8}$ https: //riha.eesti.ee/riha/onto/ettevotlus.ariregister/2010/r2
} 


\section{3 (Re)usable Knowledge Resources}

Reusable knowledge resources are formed from general and specific domain knowledge, non-ontological and ontological resources. Reuse of these resources speeds up ontology development process. Non-ontological resources are conceptual schemas of databases, glossaries, vocabularies, thesauri, regulatory documents of a state IS, descriptions of database objects, databases, service descriptions, etc. Ontological resources are existing ontologies in RDF or OWL format available at ontology libraries or repositories. For example, domain ontologies of state IS collected to RIHA repository could be reused for construction of a new ontology in ontology network.

\subsection{Sequence of Activities}

Sequence of ontology development activities is depicted in Figure 1.

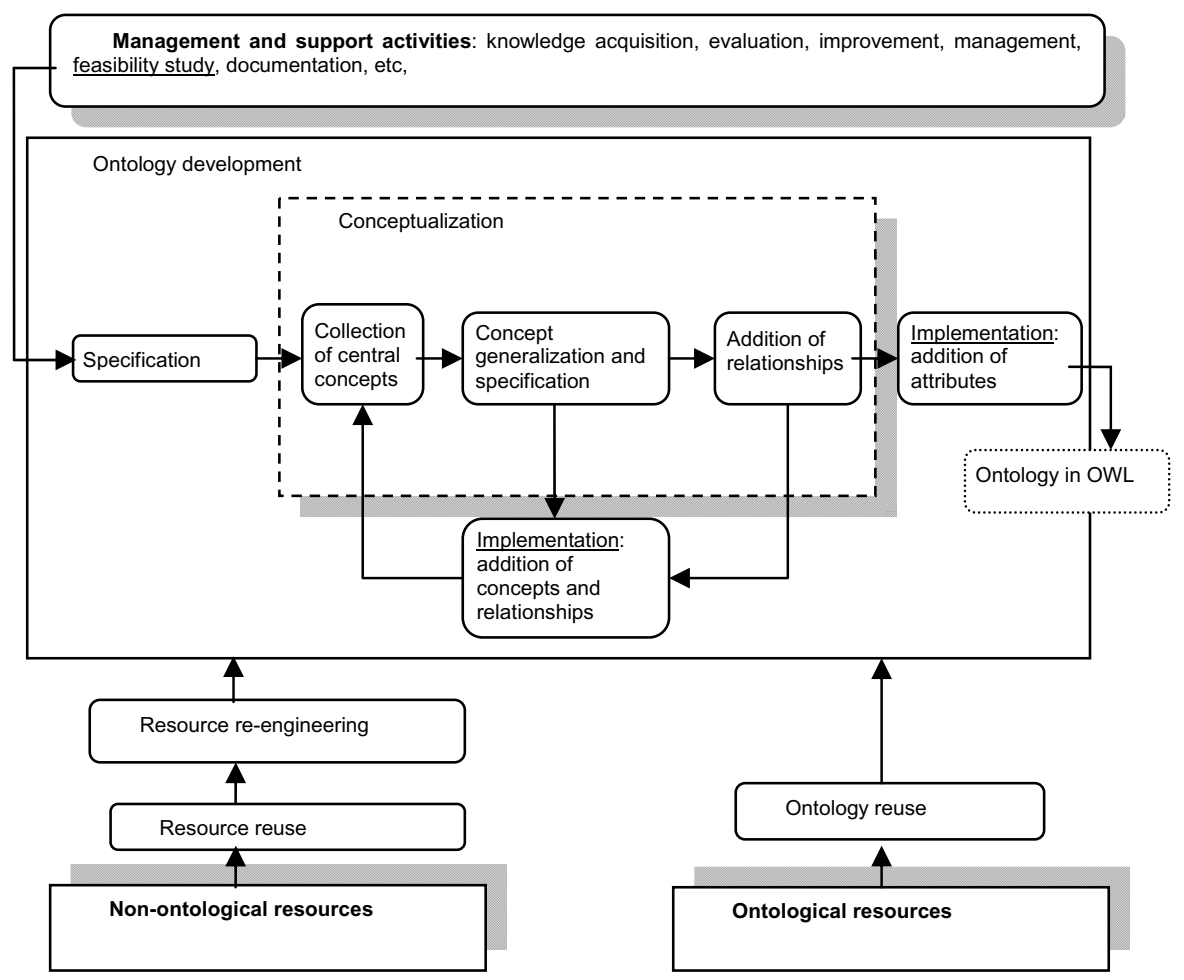

Fig. 1. Development process of domain ontologies for state IS: sequence of activities

Ontology management and support activities are performed in parallel with other ontology development activities during the whole ontology development process. 
Figure 1 shows also relationships of non-ontological and ontological resources with development process through corresponding activities.

Main activities of ontology development process are given in the Figure 1 as the following sequence: specification, conceptualization and implementation. According to iterative life cycle model early implementation of domain concepts and relationships is foreseen. At the first iteration, basic (central) domain concepts and relationships are arranged to ontological structure and implemented. After that, during each of the iterations, new concepts and relationships are added and implemented. Attributes of individuals of concepts are added in the final stage. After that ontology is evaluated according to the requirements e.g. are all input/output parameters of web services covered by corresponding components of respective domain ontology? If not, then missing components need to be added.

In the following sections the ontology development process and corresponding activities are provided in detail.

\section{Management and Support Activities}

Management and support activities are similar to those defined in METHONTOLOGY methodology [4]. Ontology development starts with activity of knowledge acquisition that will continue during the whole development process. Most of the knowledge will be acquired in the beginning of the process; needed amount of new knowledge is declining according to the development process proceeds.

\section{Reuse of Resources}

We consider ontological and non-ontological resources as stated above. In the development process of state IS domain ontologies both types of resources could be used. Reuse of non-ontological resources is most important at the stages of ontology specification and conceptualization. Reuse of ontological resources is not possible only during specification and conceptualization but also during the implementation activity. For example, ontology import is a part of implementation of ontology.

Resource reuse activities are dependent on its type as follows.

In case of non-ontological resources first resource reuse is performed and then resource reengineering activity is carried out. For example, a domain model of an IS can be easily used for acquiring possible classes to be included to corresponding domain ontology. Descriptions of data objects from the corresponding XMI file can be reused for defining data type properties for individuals of classes.

In the process of non-ontological resource (e.g. database schema) reuse the resource is analyzed with respect to its usability for ontology creation. If reusable resources are found, then reengineering of these resources is carried out. This is the process of transforming an existing non-ontological resource (e.g. data base schema, database etc.) into ontology that can be reused in the process of ontology engineering of current domain ontology.

In case of ontological resources, reengineering of some existing ontology is performed. An example is geometry ontology that can be reused in a number of ontologies related to the domain of land IS-s. 
Process of ontology reuse can be divided into the following activities:

1. Retrieval of possible candidate ontologies from public ontology repositories like Swoogle $^{9}$ and WATSON ${ }^{10}$. Ontologies developed for Estonian state IS can be retrieved from RIHA $^{11}$.

2. Evaluation of retrieved candidate ontologies from the point of view of requirements (content, language, formal representation, documentation, quality, etc).

3. Choice of suitable ontology

4. Deciding a way how ontology is to be reused. Ontology can be reused as it is. Ontology might need reengineering before it can be reused. Ontology reengineering is the process during what its conceptual model is transformed into a new conceptual model that meets requirements of a new ontology and which is then implemented. Ontology could also be merged to a new ontology.

\section{Specification}

In parallel with knowledge acquisition a set of requirements that ontology should meet are to be defined. In the process of specification activity, goal and scope of ontology, resources, and use cases are specified. In addition, a list and descriptions of central domain concepts are provided. For the development process specification helps to stay within the frame of the scope of the problem. For third parties, ontology specification serves as an initial overview of ontology.

\section{Conceptualization and an Early Implementation}

Ontology specification activity is followed by ontology conceptualization. During the conceptualization, a conceptual model of ontology that represents domain concepts and relationships between them is created. The conceptual model of ontology should take into account that ontology will be later implemented in OWL language. Ontology conceptualization is carried out according to acquired knowledge of a domain.

Ontology conceptualization is followed by ontology implementation activity resulting in ontology description of OWL language. In provided methodology ontology conceptualization and implementation are considered together because iterative life cycle model of ontology development is applied. Therefore, we call implementation stage here as an early implementation.

Different strategies could be used for conceptualization as follows:

- Top-down strategy starts from most general domain concepts and step-by-step moves to specific concepts.

- Middle out strategy proposes at first to concentrate to a set of basic (central) concepts of a domain and then to move to the direction of their generalization and specialization.

- Buttom-up strategy tries to generalize meaning of data fields related to an application/service.

\footnotetext{
${ }^{9}$ Swoogle; http: //swoogle.umbc.edu/

${ }^{10}$ Watson; http: / /watson. kmi.open.ac.uk/WatsonWUI/

${ }^{11}$ RIHA; http: / / riha. eesti.ee
} 
Taking into account that domain ontologies for state IS will be created by domain experts on the basis of their data collections and corresponding IS the middle out conceptualization strategy is the most appropriate. Central concepts can be identified easily by domain experts as these are usually denoted by central terms used to talk about data collection of the corresponding IS. These terms are also used in legislation documents related to IS. Using top-down approach (from legislation terminology to data fields) creates too many concept hierarchy levels to ontology. Bottom-up method makes it hard to generalize from the level of data fields to more general concepts and therefore it takes more time to distinguish between concepts and attributes. This in turn means that development of ontology that satisfies requirements takes more time.

In proposed methodology ontology conceptualization and implementation are divided into sub-activities as follows:

1. Collection of concepts. Central concepts of a domain are identified (for example, 7-10 concepts). Purpose and scope of IS, most often used database fields and service parameters are helpful indicators for identifying central domain concepts of ontology.

2. Concept generalization and specification of one level. Not all concepts of ontology should correspond to input/output parameters of services, there could be concepts that make ontology better understandable for third parties. However, it is recommendable to keep ontology as minimal as possible with respect to its scope.

3. Identification and addition of main relationships. After central concepts and their generalizations and specifications are identified, then the main relationships between these concepts are to be added to ontology.

4. Early implementation. Result of ontology implementation is its description in OWL. Early implementation enables to detect logical errors of ontology description as well as get a view of its complexity and see how well it meets the requirements. As a rule, ontology creation tools like Protégé ${ }^{12}$ are used. These tools include built-in DL reasoners (for example, Pellet ${ }^{13}$, Fact $++{ }^{14}$ etc) in order to automatically find inconsistencies in ontology description presented in OWL.

5. Iterative development. As the main goal of ontology its to cover all concepts related to input/output parameters of services, then during consecutive iterations all service parameters will be taken into account and corresponding to them concepts are identified and step-by-step added to ontology. In case of iterative development a set of new concepts to be added to ontology is decreasing making possible to limit development process to implementation activity in the final iteration steps.

6. Addition of datatype properties. As a rule, datatype properties correspond to data objects (database fields like last name, first name, SSN, etc) of a given state IS and are added to the ontology and implemented during final iteration of its development.

\footnotetext{
${ }^{12}$ Protégé; http: / / protege. stanford.edu/

${ }^{13}$ Pellet; http: / /www. clarkparsia.com/pellet

${ }^{14}$ Fact++; http : / / owl . man.ac.uk/ factplusplus
} 
7. Annotation of ontology components. After logical consistency of ontology is proved ontology components are to be annotated (e.g. descriptions of natural language(s) are added to components). It is user-friendly to provide a documentation of ontology in addition to its description in the form of machinereadable OWL file. This makes it easier to reuse, maintain and further development of ontology.

In order to create conceptual model of ontology it is recommended to use some method of knowledge organization and structuring. Glossary of terms and concepts, concept taxonomy, list of relationships between concepts and attributes of individuals could serve for this purpose. Corresponding activity could be called as documentation of conceptualization of ontology.

\section{$5 \quad$ Evaluation of the Methodology}

The provided methodology has been iteratively developed and evaluated during the last 2 years within the framework of achieving semantic interoperability of state IS in Estonia. The methodology was widely used in numerous training courses on ontology engineering provided for domain experts of state IS-s. The feedback from participants of courses was used to improve the methodology in order to meet needs and take into account background knowledge of domain experts. By now, the methodology is accepted as the ontology development methodology for creation of domain ontologies of state IS in Estonia. During the year 2010, 21 domain ontologies of state IS were developed using this methodology. The complexity of ontologies required for achieving semantic interoperability of state IS as already mentioned is not very high. Domain ontologies of state IS are intended to be used for semantic annotation of web services and as a rule do not contain descriptions of defined classes. The following table 1 represents some quantitative characteristics of a few typical state IS domain ontologies.

Table 1. Ontology metrics of some state IS ontologies

\begin{tabular}{lccccl}
\hline Name of ontology & $\begin{array}{c}\text { Count } \\
\text { of } \\
\text { classes }\end{array}$ & $\begin{array}{c}\text { Object } \\
\text { properties }\end{array}$ & $\begin{array}{c}\text { Data-type } \\
\text { properties }\end{array}$ & $\begin{array}{c}\text { Subclass } \\
\text { axioms }\end{array}$ & DL expressivity \\
\hline $\begin{array}{l}\text { Address objects } \\
\begin{array}{l}\text { Central commercial } \\
\text { register }\end{array}\end{array}$ & 101 & 13 & 48 & 17 & ALCQ(D) \\
$\begin{array}{l}\text { Mineral deposits } \\
\begin{array}{l}\text { Estonian } \\
\text { topographic domain }\end{array}\end{array}$ & 25 & 19 & 133 & 12 & ALCQ(D) \\
$\begin{array}{l}\text { Estonian geodetic } \\
\text { domain }\end{array}$ & 169 & 9 & 39 & 158 & ALCRQ(D) \\
\hline
\end{tabular}


It was intention from the very beginning of the ontology development for state IS to keep ontologies as simple as possible in order to make it possible to domain experts to create ontologies of their respective fields. Some of ontologies are not entirely finalized as more datatype properties are to be added in order to meet requirements of semantic annotations of web services.

According to the law, domain ontologies are to be collected to RIHA repository. However, not all of them are there. In 2010, only 7 ontologies were published. A quality assurance procedure of ontologies to be stored in RIHA has put in place and before ontology does not meet respective requirements, it is not published in RIHA.

The methodology proposed in this paper aimed at development of ontology network of state IS domain ontologies. With this respect RIHA serves as ontology repository for ontology reuse and re-engineering. The SEHKE tool [15] is intended to support collaborative ontology development and managing network of ontologies.

State IS in Estonia has according to RIHA over 20000 data objects. These should be annotated using corresponding ontology components (i.e. datatype properties). Consequently, the ontology network should contain approximately the same number of datatype properties in addition to concepts and object properties. By now, we have about 500 datatype properties defined in our current version of the ontology network. According to this, we are in the beginning of development of ontology network of state IS in Estonia.

These data objects are used by about 1500 X-road services (machine-to-machine data services) which input/output parameters should be semantically annotated. This process has just started.

\section{Conclusion}

Demand for state IS domain ontologies to be used for solving semantic interoperability problems of e-government services requires easier process of creating ontologies than general technically complex ontology development methodologies provide. In this paper we presented a novel ontology development methodology for domain experts who being actual domain knowledge holders easily identify central domain concepts and using early implementation and iterative development activity could build ontologies without need for ontology engineer. The approach is evaluated by development of network of e-government domain ontologies of state IS in Estonia.

Lessons learned from the use of the methodology show that implementation activity is sometimes still too complex for domain experts even if they use easy-to-use ontology editors. Therefore, the future work is planned to make this ontology development methodology even easier by using simple intermediate representations of domain conceptualization that can be automatically translated to OWL ontology. In this case, implementation activity of provided methodology will be automatic or will be at least semi-automatic.

Acknowledgments. This research was partially supported by the target-financed theme No. 0322709s06 of the Estonian Ministry of Education and Research as well as 
by the ERDF funded Estonian Centre of Excellence in Computer Science, EXCS. I am particularly grateful to Leive Turi for creating the first ontology using this methodology and collaborating to the development of the methodology.

\section{References}

1. Barzdins, J., Barzdins, G., Balodis, R., Cerans, K., Kalnins, A., Opmanis, M., Podnieks, K.: Towards Semantic Latvia. In: Proceedings of Communications of 7th International Baltic Conference on Databases and Information Systems, Vilnius, Lithuania, pp. 203-218 (2006),

http://melnais.mii.lu.lv/audris/CReady_Barzdins_TowardsSeman ticlatvia.pdf

2. Bednár, P., Furdík, K., Paralič, M., Sabol, T., Skokan, M.: Semantic Integration of Government Services - the Access-eGov Approach. In: Cunningham, P., Cunningham, M. (eds.) Collaboration and the Knowledge Economy: Issues, Applications, Case Studies. IOS Press, Amsterdam (2008)

3. Georgiadis, P., Lepouras, G., Vassilakis, C., Boukis, G., Tambouris, T., Gorilas, S., Davenport, E., Macintosh, A., Fraser, J., Lochhead, D.: SmartGov: A Knowledge-based Platform for Transactional Electronic Services. Springer, Heidelberg (2002)

4. Gómez-Pérez, A., Fernández-López, M., Corcho, O.: Ontological Engineering with examples from the areas of Knowledge Management, e-Commerce and the Semantic Web. Springer, Heidelberg (2004)

5. Haase, P., Rudolph, S., et al.: NeOn Deliverable D1.1.1 Networked Ontology Model (November 2006), http: / / www. neon-project.org/

6. Haav, H.-M., Kalja, A., Küngas, P., Luts, M.: Ensuring Large-Scale Semantic Interoperability: The Estonian Public Sector's Case Study. In: Haav, H.-M., Kalja, A. (eds.) Databases and Information Systems V, pp. 117-129. IOS Press (2009)

7. Hyvönen, E., Viljanen, K., Tuominen, J., Seppälä, K.: Building a National Semantic Web Ontology and Ontology Service Infrastructure -The FinnONTO Approach. In: Bechhofer, S., Hauswirth, M., Hoffmann, J., Koubarakis, M. (eds.) ESWC 2008. LNCS, vol. 5021, pp. 95-109. Springer, Heidelberg (2008),

http: / /www. seco.tkk/publications/2008/hyvonen-et-albuilding-2008.pdf

8. IEEE Standard for Developing Software Life Cycle Processes. IEEE Std 1074-1997

9. Kalja, A.: New version of the x-road. In: Information Society Yearbook 2009, Ministry of Economic Affairs and Communications of Estonia, Department of State Information Systems (RISO), Tallinn (2010), http: / /www.riso.ee/en/pub/2009it/\#p=2 $1-4$

10. Lavbiĉ, D., Krisper, M.: Rapid Ontology Development. In: Welzer Druzovec, T., et al. (eds.) Proceeding of the 2010 Conference on Information Modelling and Knowledge Bases XXI, pp. 283-290. IOS Press (2010)

11. Loutas, N., Tarabanis, V.P.: Providing public services to citizens at the national and paneuropean level using semantic web technologies. In: Proceedings of the Sixth Eastern European eGov Days, Czech Republic (2008)

12. Parmakson, P., Vegmann, E.: The administration system of the state information system (RIHA). In: Information Society Yearbook 2009, Ministry of Economic Affairs and Communications of Estonia, Department of State Information Systems (RISO), Tallinn (2010), http: / / www.riso.ee/en/pub/2009it/\#p=2-1-6 
13. Staab, S., Schnurr, H.P., Studer, R., Sure, Y.: Knowledge Processes and Ontologies. IEEE Intelligent Systems 16(1), 26-34 (2001)

14. Stojanovic, L., Kavadias, G., Apostolou, D., Probst, F., Hinkelmann, K.: Ontology-enabled e-Gov Service Configuration, Deliverable Ontogov Project - IST Project 507237, http: / / www . ontogov. com

15. Tomingas, K., Luts, M.: Semantic Interoperability Framework for Estonian Public Sector's E-Services Integration. In: d'Aquin, M., García Castro, A., Lange, C., Viljanen, K. (eds.) ESWC 2010/ORES 2010 Proceedings of the First Workshop on Ontology Repositories and Editors for the Semantic Web (ORES 2010) 7th Extended Semantic Web Conference Hersonissos, Crete, Greece (May/June 2010)

16. Uschold, M., Grueninger, M.: Ontologies: principles, methods and applications. Knowledge Sharing and Review 11 (1996)

17. Zhou, L., Booker, Q., Zhang, D.: Toward Rapid Ontology Development for Underdeveloped Domains. In: Proceedings of the 35th Annual Hawaii International Conference on System Sciences (HICSS 2002), vol. 4, pp. 106-115 (2002) 